\title{
Treatment of Recalcitrant Plantar Wart Unresponsive to Cryotherapy with Acupuncture and Moxibustion: A Case Report
}

\author{
Soo Bo Shim ${ }^{1}$, Hye Lim Lee ${ }^{1}$, and Hyun Kyung Sung ${ }^{1}$ \\ ${ }^{1}$ Affiliation not available
}

January 20, 2021

\begin{abstract} for plantar warts in the paediatric patient population. A Case Report

Soo Bo Shim ${ }^{1}$, Hyun Kyung Sung ${ }^{2 *}$, Hye Lim Lee ${ }^{1 *}$

${ }^{1}$ Department of Pediatrics, College of Korean Medicine, Daejeon University

${ }^{2}$ Department of Pediatrics, College of Korean Medicine, Semyung University
\end{abstract}

This study presents the case of a 9-year-old girl for whom complete resolution of recalcitrant warts was achieved with acupuncture and moxibustion treatment. This result suggests that acupuncture and moxibustion could be effective therapeutic strategies

Treatment of Recalcitrant Plantar Wart Unresponsive to Cryotherapy with Acupuncture and Moxibustion:

Author information

Soo Bo Shim; Department of Korean Pediatrics, Daejeon Korean Medicine Hospital of Daejeon, 75, Daedeokdaero 176beon-gil, Seo-gu, Daejeon, Republic of Korea; Tel: +82-42-470-9138 / Fax: +82-42-477-9007; sbopink@gmail.com

\section{Corresponding Author}

Hyun Kyung Sung; Department of Korean Pediatrics, Semyung University, St. Semyung 65, Jecheon, Republic of Korea; Tel: +82-43-5841-1739 / Fax: +82-43-856-1731;shksolar@gmail.com

Hye Lim Lee; Department of Korean Pediatrics, Daejeon Korean Medicine Hospital of Daejeon, 75, Daedeok-daero 176beon-gil, Seo-gu, Daejeon, Republic of Korea; Tel: +82-42-470-9138 / Fax: +82-42477-9007;hanilim03@gmail.com

\section{Abstract}

This study presents the case of a 9-year-old girl for whom complete resolution of recalcitrant warts was achieved with acupuncture and moxibustion treatment. This result suggests that acupuncture and moxibustion could be effective therapeutic strategies for plantar warts in the paediatric patient population.

Keywords : Recalcitrant warts, Plantar warts, Acupuncture, Moxibustion

Background

Warts are a common viral skin disease, caused by human papillomavirus infection ${ }^{1}$, having high prevalence rates ranging between $5 \%$ and $30 \%$ in children ${ }^{2}$. There are numerous treatments for warts, such as liquid nitrogen, cryotherapy, and excision. Liquid nitrogen is the most frequently used treatment for warts; however, 
the treatment procedure is generally very painful ${ }^{3}$. Curettage, topical salicylic acid, and cryotherapy are often used for plantar warts ${ }^{4}$. Excision is not recommended, as it leads to frequent recurrences or scarring ${ }^{4}$. Furthermore, in the case of therapy-resistant lesions, laser and photodynamic therapy approaches could be used $^{5}$ but these methods are time-consuming and costly.

Therefore, in the case of plantar warts, both first- and second-line treatments are generally still unsatisfactory due to their low efficacy, low local tolerability, and high recurrence rate $^{6}$.

Acupuncture is the insertion of fine needles into specific body sites ${ }^{7}$. It has been used successfully to treat acute skin eruptions, acne, atopic dermatitis, psoriasis, warts, and leg ulcers ${ }^{8,9}$. Meanwhile, moxibustion is a procedure that uses the heat generated by the burning of herbal preparations to stimulate specific points for treating disease ${ }^{10}$. It is used as a treatment for a wide range of conditions, including osteoarthritis, breech presentation, supportive cancer care, and cutaneous warts ${ }^{11}$. Recent research has shown that moxibustion is effective in the treatment of cutaneous warts, and that acupuncture is an effective treatment for HPV-related warts through the modulation of the immune system. However, the use of acupuncture and moxibustion in the treatment of recalcitrant plantar warts has not been reported until now. We report a case of recalcitrant plantar wart successfully resolved after acupuncture and moxibustion treatment.

Case presentation

A 9-year-old healthy Korean girl presented to our department with recalcitrant plantar warts on her soles, that had been present for over 6 months and had failed to respond to cryotherapy. She complained of pain while walking, standing, and even wearing shoes. Physical examination revealed that the lesions were hyperkeratinized and filled with punctuate black dots. The largest lesion measured $10 \times 15 \mathrm{~mm}$ (Fig. 1). She had no significant past medical or medication history.

She received acupuncture treatment using hand acupuncture needles $(0.18 \mathrm{~mm} \times 8.0 \mathrm{~mm}$ stainless steel needle, Dongbang Inc., Chungnam, Korea) and moxibustion treatment using mini moxa cones made of Artemisia argyi (Dongbang Inc., Chungnam, Korea). Acupuncture therapy was directly provided at all the wart lesions. Moxibustion was placed directly on the lesions, when $80 \%$ of moxa cone had burnt off and the patient could feel the burning, the moxa cone was removed with forceps, and another cone was placed. We repeated this sequence two times on all lesions within a single session.

Initially, acupuncture and moxibustion sessions were difficult to conduct, as the patient expected that she would feel pain, but we could proceed with the treatment after explaining the need for treatment and obtaining consent from the patient. At the 8th week of treatment, a burn occurred on the left toe during moxibustion treatment; it was treated with a dressing and followed up at every visit.

After 12 weeks of treatment, the hyperkeratotic lesion and black dots disappeared (Fig. 2.). Eight weeks later, the lesions and pain were almost completely resolved (Fig. 3). At the 3-month follow-up, the lesions remained fully resolved (Fig. 4,5). The patient stated that the discomfort felt while wearing shoes had disappeared after the treatment, and she was satisfied with the treatment.

Discussion and Conclusion

In this case report, we demonstrated the use of acupuncture and moxibustion in the treatment of therapyresistant plantar warts, with a high clinical efficacy. It is remarkable that complete clearance of the wart lesion was achieved with a 20 -week treatment without any pre-treatment curettage or additional treatments.

Warts have high prevalence rates ranging between $5 \%$ and $30 \%$ in children ${ }^{2}$. Among them, plantar warts account for $30 \%$ of all cutaneous warts ${ }^{12}$. Spontaneous resolution of warts within 2 years is common in children. However, recalcitrant cases can cause considerable morbidity ${ }^{13}$.

There are numerous treatments for warts, including destructive therapy, virucidal therapy, antimitotic therapy, and immunotherapy. Epidermal damage can be produced by chemical means, such as salicylic acid, or by physical means, including cryotherapy, lasers, photodynamic therapy, or hyperthermia ${ }^{14}$. However, some treatment could be very painful with a long downtime posttreatment period, and the treatment of plantar 
wart remains a challenge ${ }^{1}$. Therefore, acupuncture and moxibustion treatment are being studied as alternative therapies. In 2016, Yun et al. reported that 19-22 weeks of moxibustion treatment can effectively eliminate warts ${ }^{11}$. According to Brustin et al., the therapeutic effect of acupuncture on persistent warts is related to the immune response and anti-inflammatory effects ${ }^{15}$. Performing acupuncture and moxibustion therapy for children is difficult because they are afraid of the treatment, and there is a risk of burns. Therefore, better measures should be explored to ensure treatment compliance in children.

The limitation of this case report is that it only dealt with a single case, and that burns occurred during moxibustion. However, this case report suggests that acupuncture and moxibustion could be effective therapeutic strategies in subjects with "difficult-to-treat" plantar wart lesions.

This report represents a single case study of a recalcitrant plantar wart treated successfully using acupuncture and moxibustion directly on the lesions. Further studies are required to fully assess the effectiveness and safety of this therapy.

\section{Ethics approval and consent to participate:}

The patient consent was obtained by patient's caregiver during the treatment period and this study was confirmed by the Daejeon Korean Medicine Hospital of Daejeon University Institutional Review Board (IRB)(DJDSKH-20-E-23).

\section{Availability of data and materials:}

Data will be made available upon request.

\section{Competing interest:}

The authors declare that they have no competing interests.

\section{Funding:}

This study was supported by the Semyung University Research Grant of 2018.

\section{Authors' contributions:}

SBS and HLL wrote the original draft. HKS provided the financial support. HLL and HKS critically review and revised the paper. All authors approved the final version.

\section{Acknowledgements:}

Not applicable.

\section{References}

1. Meloni G, Milani M. Efficacy and tolerability of topical green tea extract (Polyphenon E) application in a "therapy-resistant" plantar wart. Case Rep Dermatol . 2018;10(2):127-32.

2. van Haalen FM, Bruggink SC, Gussekloo J, Assendelft WJ, Eekhof JA. Warts in primary schoolchildren: prevalence and relation with environmental factors. Br J Dermatol . 2009;161(1):148-52.

3. Gibbs S, Harvey I, Sterling J, Stark R. Local treatments for cutaneous warts: systematic review. BMJ . 2002;325:461.

4. Sterling JC Handfield-Jones S, Hudson PM, Dermatologists. BAo. Guidelines for the management of cutaneous warts. Br J Dermatol.2001;144:4-11.

5. Fuchs SM, Fluhr JW, Bankova L, Tittelbach J, Hoffmann G, Elsner P. Photodynamic therapy (Pdt) and waterfiltered infrared a (Wira) in patients with recalcitrant common hand and foot warts. Ger Med Sci . 2004;2:8.

6. Kodner CM, Nasraty S. Management of genital warts. Am Fam Physician . 2004;70:2335-42. 
7. Shakibapour M, Mahmoodi M, Hoseini SG, Rostami F, Mansurian M, Jafari R, et al, Hoseini SG, et al. Therapeutic effect of acupuncture in Balb/C model of cutaneous leishmaniasis. Adv Biomed Res . 2015;4:77.

8. Rosted P. Treatment of skin diseases with acupuncture: a review.J Dermatol Treat. 1995;6:241-2.

9. Baron SE, Goodwin RG, Nicolau N, Blackford S, Goulden V. Use of complementary medicine among outpatients with dermatologic conditions within Yorkshire and South Wales, United Kingdom. J Am Acad Dermatol . 2005;52:589-94.

10. Lee MS,Kang JW, Ernst E. Does moxibustion work? An overview of systematic reviews. BMC ResNotes . 2010;3:284.

11. Yun Y, Shin S, Kim KS, Ko SG, Choi IW. Three cases of cutaneous warts treated with moxibustion. Explore (NY). 2016;12(4):277-81.

12. Kilkenny M, Marks R. The descriptive epidemiology of warts in the community. Australas J Dermatol. $1996 ; 37(2): 80-6$.

13. Abeck D Folster-Holst R. Quadrivalent human papillomavirus vaccination: a promising treatment for recalcitrant cutaneous warts in children. Acta Derm Venereol . 2015;95:1017-9.

14. Sterling JC, Gibbs S, Hussain S, Mustapa M, Handfield-jones S. British Association of Dermatologists' guidelines for the management of cutaneous warts. Br J Dermatol . 2014;171(4):696-712.

15. Brustin R, Toledano M, Geffen T, Goona R, Hochberg M, Kerisberg B, et al. Immune modulation and treatment of human papilloma virus-related warts with energetics of living systems acupuncture. Med Acupunct . 2017;29(3):145-54.

\section{Figure Legends}

Fig. 1. Lesions at first visit

Fig. 2. Lesions at 12 weeks after treatment

Fig. 3. Lesions at 20 weeks after treatment

Fig. 4. Lesions at 3-month follow-up

Fig. 5. Wart treatment timeline 


$$
\frac{80}{20}
$$



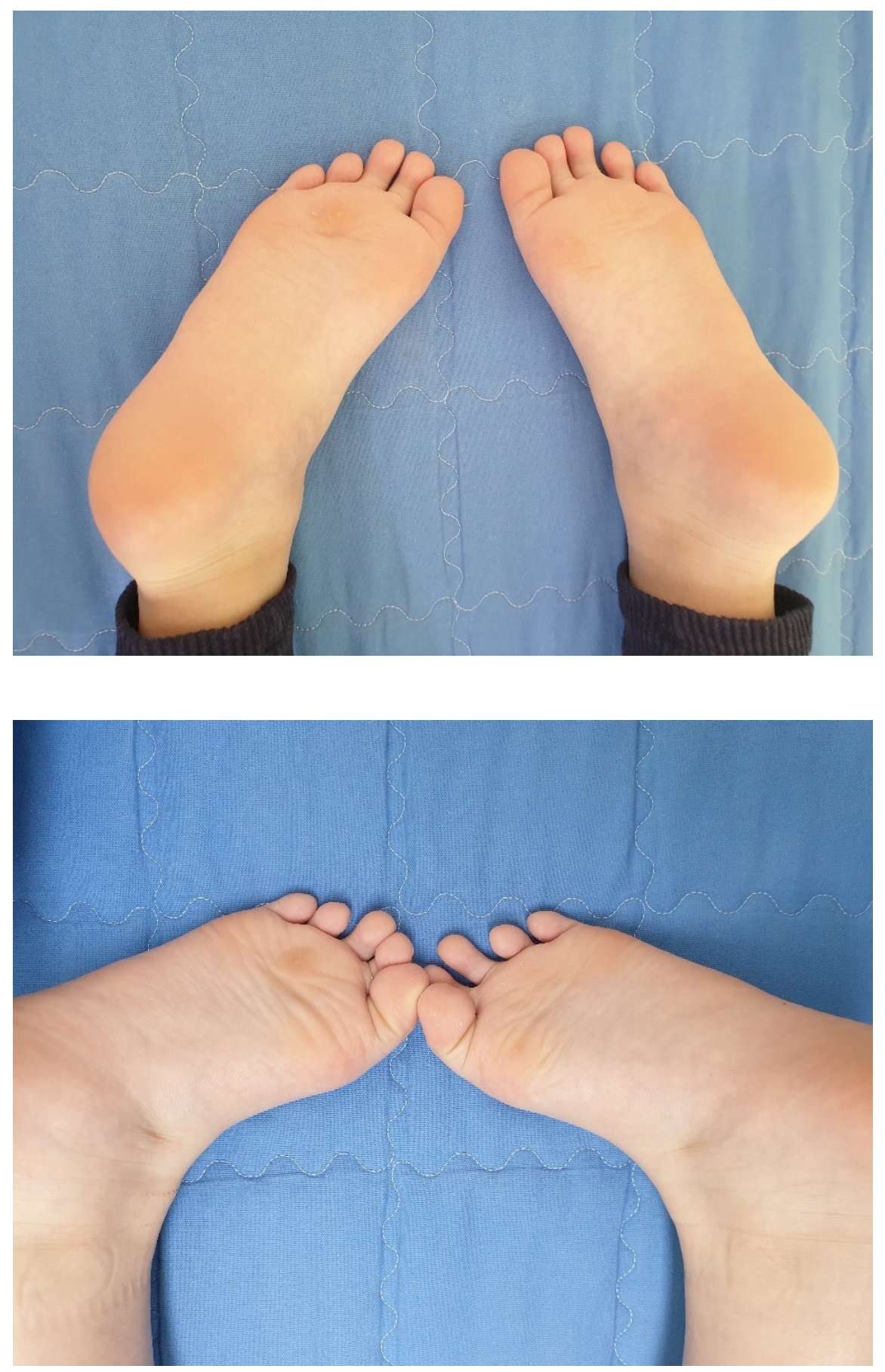


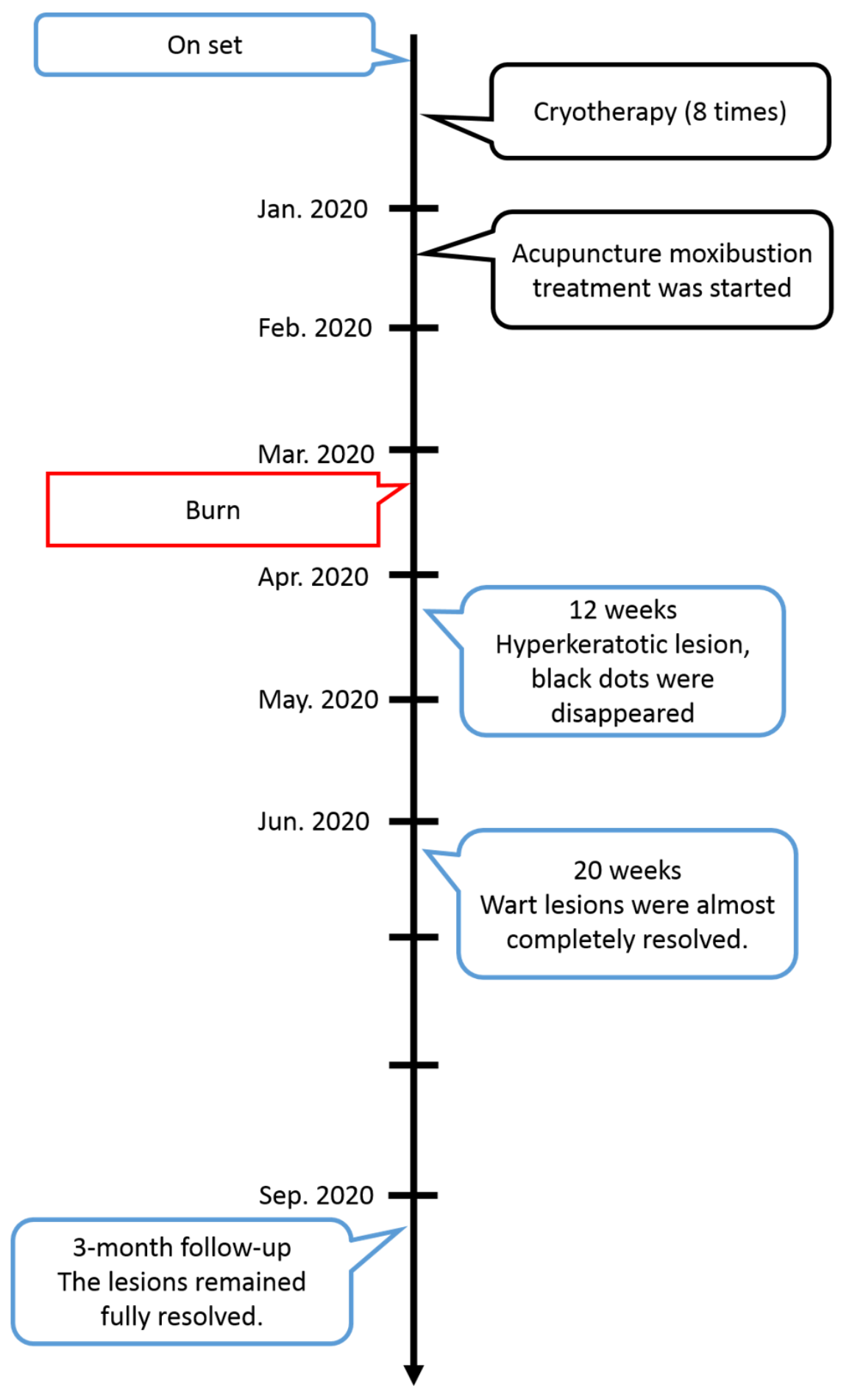

